\title{
Entropy-Expansiveness and Domination for Surface Diffeomorphisms
}

\author{
María José PACIFICO and José L. VIEITEZ \\ Instituto de Matematica \\ Universidade Federal do Rio de Janeiro \\ C. P. 68.530 , CEP 21.945-970 \\ Rio de Janeiro, R. J. - Brazil \\ pacifico@im.ufrj.br \\ Instituto de Matematica \\ Facultad de Ingenieria \\ Universidad de la Republica \\ CC30, CP 11300 Montevideo - Uruguay \\ jvieitez@fing.edu.uy
}

Received: July 3, 2006

Accepted: March 10, 2007

\begin{abstract}
Let $f: M \rightarrow M$ be a $C^{r}$-diffeomorphism, $r \geq 1$, defined on a closed manifold $M$. We prove that if $M$ is a surface and $K \subset M$ is a compact invariant set such that $T_{K} M=E \oplus F$ is a dominated splitting then $f / K$ is entropy expansive. Moreover $C^{1}$ generically in any dimension, isolated homoclinic classes $H(p)$, $p$ hyperbolic, are entropy expansive.

Conversely, if there exists a $C^{1}$ neighborhood $\mathcal{U}$ of a surface diffeomorphism $f$ and a homoclinic class $H(p), p$ hyperbolic, such that for every $g \in \mathcal{U}$ the continuation $H\left(p_{g}\right)$ of $H(p)$ is entropy-expansive then there is a dominated splitting for $f / H(p)$.
\end{abstract}

Key words: entropy-expansiveness, homoclinic classes, dominated splitting, homoclinic tangency, symbolic extension.

2000 Mathematics Subject Classification: 37D30, 37C29, 37E30.

\section{Introduction}

Since the seminal work of Smale [37] establishing the main goals to describe the long term evolution of discrete or continuous time dynamical systems, one of the main strategies has been to prescribe some property at the infinitesimal level of the system that implies a definite behavior for the underlined dynamics. Examples include the concepts of hyperbolicity, partial hyperbolicity and dominated splitting. In the hyperbolic case the tangent bundle of an invariant set $K, T_{K} M$ splits into two 
complementary directions $S \oplus U$, invariant by the action of the differential map, $S$ exponentially contracted and $U$ exponentially expanded by this action. In the case of partial hyperbolicity one of these directions, for instance the contracting one $S$, is replaced by the direct sum of a contracting and a neutral direction $S \oplus C$. In the case of a dominated splitting it holds that $T_{K} M=E \oplus F$ where $E$ and $F$ are invariant by the action of the differential map. Albeit nothing can be said about the size of forward iterated vectors by the differential map, it can be assured that the direction of vectors in $T_{K} M \backslash E$ converges exponentially fast to $F$. We have

hyperbolicity $\Longrightarrow$ partial hyperbolicity $\Longrightarrow$ existence of a dominated splitting.

But the converse is not generally true.

These concepts allow to understand large classes of dynamics, particularly those exhibiting sensibility to initial data and chaos. Systems having these properties constitute an active area of research in Dynamical Systems.

On the other hand, one may also ask what are the consequences at the infinitesimal level from a known behavior of the evolution system at the ambient manifold, in particular we wish to be able to say when a dynamical system is (partially) hyperbolic or has a dominated splitting. But rarely a property displayed by a system solely implies a distinguishing behavior of the differential map acting at the tangent bundle. For instance, in $[17,18]$ it is proved that a (generalized) pseudo-Anosov map $f$ is ergodic and even Bernoulli. Thus they have very rich properties from the point of view of chaotic dynamics. But for those maps there is at least a periodic point $p$ where the derivative $D f_{p}$ is idempotent and so the dynamics at the tangent bundle level cannot be characterized in terms of hyperbolicity, partial hyperbolicity or even the existence of a dominated splitting. On the other hand under an arbitrarily small $C^{1}$ perturbation of $f$ we may create sinks or sources hence loosing ergodicity. Example 1.1 is a generalized pseudo-Anosov map defined on $S^{2}$ illustrating such a behavior.

Hence, it is natural to ask, for a given $C^{r}$ topology defined in $\operatorname{Diff}^{s}(M), r, s \geq 1$, which robust properties satisfied by the underlined system have dynamical consequences at the tangent bundle level and vice versa. Several authors have worked in this line of ideas (see, for instance, $[13,23,25,30,35]$ ), particularly in the $C^{1}$ topology. Here by a robust property we mean a property shared by all system in a $C^{r}$-neighborhood of the original one.

Answers to these questions when restricted to homoclinic classes $H(p)$ associated to a hyperbolic periodic point $p$ of $f$ would give more information about these sets. Recall that the homoclinic class $H(p)$ of a saddle-type hyperbolic periodic point $p$, is the closure of the transverse intersections of the stable manifold $W^{s}(p)$ with the unstable manifold $W^{u}(p)$ of $p$. Points $x \in W^{s}(p) \cap W^{u}(p), x \neq p$, are called homoclinic points. They are characterized by the fact that both forward and backward orbits converge to the orbit of the periodic point $p$. Their importance was first realized by Poincaré when studying Celestial Mechanics, mainly in the three body problem. Poincaré observed that for such a system (i) there can exist homoclinic points, (ii) the 
existence of a single homoclinic point forces the existence of infinitely many of them, and (iii) the appearance of chaotic motions.

Homoclinic classes are, in the hyperbolic case, the elementary pieces (basic pieces) of the dynamics, i.e., maximal invariant hyperbolic sets. Thus in the hyperbolic case different homoclinic classes are disjoint. Moreover, these properties were proved to be true for a residual subset of $C^{1}$ diffeomorphisms on any $n$-dimensional manifold [9].

In [2] it is proved that for generic $C^{1}$ diffeomorphisms, the elementary pieces are the chain recurrent classes, and when one of these sets contains a periodic point $p$ it coincides with the homoclinic class of $p$. Moreover, [9] together with the Closing Lemma of [32], give that the homoclinic classes constitute a partition of a dense part of the limit set of generic diffeomorphisms, and [10] establishes that chain recurrent sets of generic diffeomorphisms are Hausdorff limits of homoclinic classes. All these results evidence the importance of understanding the dynamics restricted to homoclinic classes. For more on this, and a discussion of the notion of elementary pieces of dynamics, see [5] and the references therein.

The facts described above motivate our interest in the description of the dynamics of homoclinic classes, especially in the non-hyperbolic context. We note that every homoclinic class is $f$-invariant and transitive. However, a homoclinic class may be robustly non-uniformly hyperbolic: it contains in a robust way hyperbolic saddles with different indices from $p$, see $[11,12,14]$ for constructions illustrating this fact.

One weak form of hyperbolicity in $H(p)$ can be expressed in terms of expansiveness: $f$ is $\alpha$-expansive in $H(p)$ if $\operatorname{dist}\left(f^{n}(x), f^{n}(y)\right) \leq \alpha$ for all $n \in \mathbb{Z}$, with $x, y \in H(p)$, implies $x=y$. It is well known that hyperbolicity implies $\alpha$-expansiveness for some $\alpha>0$. But expansiveness alone does not guarantee hyperbolicity, see [30, section 2]. However, if expansiveness holds in a robust way, then generically, for 3-dimensional diffeomorphisms, the homoclinic class is hyperbolic [30]. Here robust means that for all diffeomorphism $g$ that is $C^{1}$ near $f$ the homoclinic class $H\left(p_{g}\right)$ of the continuation $p_{g}$ of $p$ is $\alpha$-expansive.

This was generalized in [29] for co-dimension one homoclinic classes in two ways: First, for $n$-dimensional diffeomorphisms, robustly expansive codimension-one homoclinic classes have a codimension-one dominated splitting. Second, robustly expansive codimension-one homoclinic classes with a dominated splitting are hyperbolic.

One can relax the notion of expansiveness requiring entropy-expansiveness. This last notion is characterized by the fact that there is $\varepsilon>0$ such that for any point $x \in M, \bigcap_{n \in \mathbb{Z}} f^{n}(B(x, \varepsilon))$ is a set of topological entropy zero (see Definition A). Note that if $f$ is an expansive homeomorphism then there is a positive constant $\alpha$ such that the set $\bigcap_{n \in \mathbb{Z}} f^{n}(B(x, \alpha))=\{x\}$, which trivially has zero entropy. In this paper under the hypothesis of robust entropy-expansiveness, we study what are the consequences at the dynamical behavior of the tangent map $D f$ of a diffeomorphism $f: M \rightarrow M$. In this direction we obtain that the tangent bundle has a $D f$-invariant dominated splitting. Reciprocally, we show, in the case of surfaces, that the existence of a dominated splitting for the tangent bundle of a homoclinic class $H(p), p$ a hyper- 
bolic periodic point, implies robust entropy expansiveness for the diffeomorphism $f$ restricted to $H(p)$. Thus robust entropy expansiveness is equivalent to the existence of a dominated splitting for homoclinic classes of surfaces diffeomorphisms.

Example 1.1 exhibits a diffeomorphism $f$ that is not entropy expansive. In this case $\Omega(f)=S^{2}$ is a homoclinic class. In the example it is possible to observe what occurs for $C^{1+\sigma}$ surface diffeomorphisms when entropy expansiveness does not hold: a sequence of arbitrarily small horseshoes appears [21]. The diffeomorphism of the example is of class $C^{\infty}$ and hence it is asymptotically entropy expansive (see Definition C) by a result of Buzzi [8]. The first example of a diffeomorphism that is not entropy expansive neither asymptotically entropy expansive was given by Misiurewicz in [26], answering a question posed by Bowen [7]. Nevertheless we add our example to this article because of its nice properties: (i) it is defined on the sphere $S^{2}$ while the example of Misiurewicz is in greater dimension, (ii) it is ergodic and even Bernoulli, (iii) it admits analytic models and so there are non entropy-expansive diffeomorphisms in this setting too. Moreover, a straightforward modification of this example produces a diffeomorphism (Example 1.2) defined on a manifold of dimension greater than 2 that does have a dominated splitting defined on a homoclinic class, but it is not entropy expansive. Thus we cannot expect to generalize Theorem A directly.

A way to measure the lack of expansiveness of a non trivial homoclinic class $H(p)$ is to calculate the entropy of the set of points in $H(p)$ whose forward and backward iterates do not separate by a positive factor. Roughly speaking, if the entropy of such set is sufficiently small, almost all points of the system can be distinguished, and the homoclinic class is "almost" expansive. Again $h$-expansiveness solely does not guaranty neither hyperbolicity nor the existence of a dominated splitting for the tangent bundle, as one can see in section 1. This does not work in general. For instance, Morse-Smale diffeomorphisms are entropy expansive but are far away from being expansive. However $h$-expansiveness implies existence of a dominated splitting for non trivial homoclinic classes.

Let us now give precise definitions. Let $M$ be a compact connected boundaryless Riemannian $d$-dimensional manifold and $f: M \rightarrow M$ a homeomorphism. Let $K$ be a compact invariant subset of $M$ and dist : $M \times M \rightarrow \mathbb{R}^{+}$a distance in $M$ compatible with its Riemannian structure. For $E, F \subset K, n \in \mathbb{N}$, and $\delta>0, E$ is said to $(n, \delta)$-span $F$ with respect to $f$ if for each $y \in F$ there is $x \in E$ such that $\operatorname{dist}\left(f^{j}(x), f^{j}(y)\right) \leq \delta$ for all $j=0, \ldots, n-1$. Let $r_{n}(\delta, F)$ denote the minimum cardinality of a set that $(n, \delta)$-spans $F$. Since $K$ is compact $r_{n}(\delta, F)<\infty$. We define

$$
h(f, F, \delta) \equiv \limsup _{n \rightarrow \infty} \frac{1}{n} \log \left(r_{n}(\delta, F)\right)
$$

and the topological entropy of $f$ restricted to $F$ as

$$
h(f, F) \equiv \lim _{\delta \rightarrow 0} h(f, F, \delta) .
$$

The last limit exists since $h(f, F, \delta)$ increases as $\delta$ decreases to zero. 
For $x \in K$ let us define

$$
\Gamma_{\varepsilon}(x, f) \equiv\left\{y \in M \mid d\left(f^{n}(x), f^{n}(y)\right) \leq \varepsilon, n \in \mathbb{Z}\right\} .
$$

We will simply write $\Gamma_{\varepsilon}(x)$ instead of $\Gamma_{\varepsilon}(x, f)$ when it is understood which $f$ we refer to.

Following Bowen (see [7]) we say that

Definition A. The homeomorphism $f / K$ is entropy-expansive or $h$-expansive for short, if and only if there exists $\varepsilon>0$ such that

$$
h_{f}^{*}(\varepsilon) \equiv \sup _{x \in K} h\left(f, \Gamma_{\varepsilon}(x)\right)=0 .
$$

Definition B. If $f: M \rightarrow M$ is a $C^{s}$-diffeomorphism, $s \geq 1$, and $K \subset M$ is compact invariant, we say that $f / K$ is robustly entropy expansive if there is a $C^{1}$ neighborhood $\mathcal{U}$ of $f$ and an open set $U \supset K$ such that if $g \in \mathcal{U}$ then there is $K_{g} \subset U$ such that $g / K_{g}$ is entropy expansive. We say that $K_{g}$ is a continuation of $K$ (not necessarily unique).

Remark. We shall work in the $C^{1}$ topology because our methods to prove some of the results, as Theorem $\mathrm{C}$, involve techniques only known in this case. For instance, Hayashi Connecting Lemma [20], Pugh Closing Lemma [32], Franks Lemma [16] are valid in full generality in the $C^{1}$-topology. In fact it is known that Franks Lemma is generally false in the $C^{2}$-topology (see [34]).

The importance of $f$ being $h$-expansive is that the topological entropy can be derived from its $\varepsilon$-estimate $h(f, K, \varepsilon)$, as shown by [7, Theorem 2.4].

A similar notion to $h$-expansiveness, albeit weaker, is the notion of asymptotically $h$-expansiveness [26].

Definition C. Let $K$ be a compact metric space and $f: K \rightarrow K$ an homeomorphism. We say that $f$ is asymptotically $h$-expansive if and only if

$$
\lim _{\varepsilon \rightarrow 0} h_{f}^{*}(\varepsilon)=0 .
$$

Thus we do not require that for a certain $\varepsilon>0 h_{f}^{*}(\varepsilon)=0$ but that $h_{f}^{*}(\varepsilon) \rightarrow 0$ when $\varepsilon \rightarrow 0$. It has been proved by Buzzi that any $C^{\infty}$ diffeomorphism defined on a compact manifold is asymptotically $h$-expansive.

Next we recall the notion of dominated splitting.

Definition D. We say that a compact $f$-invariant set $\Lambda \subset M$ admits a dominated splitting if the tangent bundle $T_{\Lambda} M$ has a continuous $D f$-invariant splitting $E \oplus F$ and there exist $C>0,0<\lambda<1$, such that

$$
\left\|D f^{n}\left|E(x)\|\cdot\| D f^{-n}\right| F\left(f^{n}(x)\right)\right\| \leq C \lambda^{n} \quad \forall x \in \Lambda, n \geq 0 .
$$


Our main results are the following:

Theorem A. Let $M$ be a compact boundaryless $C^{\infty}$ surface and $f: M \rightarrow M$ be a $C^{r}$-diffeomorphism such that $K \subset M$ is a compact $f$-invariant subset with a dominated splitting $E \oplus F$. Then $f / K$ is $h$-expansive.

Since the property of having a dominated splitting is open we may conclude that any $g C^{1}$ close to $f$ is such that $g / K_{g}$ is $h$-expansive where $K_{g}$ is a continuation of $K=K_{f}$.

In case $M$ is a $d$-dimensional manifold with $d \geq 3$ the existence of a dominated splitting is not enough to guarantee $h$-expansiveness as it is shown in Example 1.2 presented below. Nevertheless a weaker result can be achieved:

Theorem B. Let $M$ be a compact boundaryless $C^{\infty}$ d-dimensional manifold and $f: M \rightarrow M$ be a $C^{r}$-diffeomorphism. Let $H(p)$ be an isolated $f$-homoclinic class associated to the $f$-hyperbolic periodic point $p$. Assume that $H(p)$ admits a dominated splitting. Then there is a $C^{1}$ neighborhood $\mathcal{U}$ of $f$ such that for a residual subset $\mathcal{R} \subset \mathcal{U}$ any $g \in \mathcal{R}$ is h-expansive when restricted to $H\left(p_{g}\right)$.

Observe that if the topological entropy of a map $f: M \rightarrow M$ vanishes, $h(f)=0$, then $f$ is $h$-expansive. For instance the identity map id : $M \rightarrow M$ is $h$-expansive. Nevertheless, robustness of $h$-expansiveness has a dynamical meaning as shows the following theorem.

Theorem C. Let $M$ be a compact boundaryless $C^{\infty}$ surface and $f: M \rightarrow M$ be a $C^{r}$-diffeomorphism. Let $H(p)$ be an $f$-homoclinic class associated to the $f$-hyperbolic periodic point $p$. Assume that there is a $C^{1}$ neighborhood $\mathcal{U}$ of $f$ such that for any $g \in \mathcal{U}$ it holds that the continuation $H\left(p_{g}\right)$ of $H(p)$ is h-expansive. Then $H(p)$ has a dominated splitting.

A natural question that arises is if Theorem $\mathrm{C}$ holds not only for surfaces but also for compact manifolds of any finite dimension. We believe that this is the case and it will be the subject of a forthcoming paper. This would imply that $C^{1}$ generically $h$-expansiveness of an isolated homoclinic class $H(p, f)$ is equivalent to the existence of a dominated splitting for $H(p, f)$.

\section{Idea of the proofs}

To prove Theorem A we proceed as follows.

- We remark that there is a compact neighborhood $U(K)$ of $K$ such that we may extend the cones defining the dominated splitting $E \oplus F$ to $U(K)$ in a continuous way.

- If $y \in M$ is such that its $f$-orbit orb $(y) \subset U(K)$ then there are defined local center stable manifolds and local center unstable manifolds $W_{\mathrm{loc}}^{\mathrm{cs}}\left(f^{n}(y)\right)$, 
$W_{\text {loc }}^{\text {cu }}\left(f^{n}(y)\right)$ for any $n \in \mathbb{Z}$. These center stable and center unstable manifolds can be obtained applying Peano's Theorem on the existence of solutions of ODE's in the plane $\mathbb{R}^{2}$.

- We choose $\varepsilon>0$ such that if $x \in K$ and $\operatorname{dist}(x, y) \leq \varepsilon$ then $y \in U(K)$ and so the local center stable and unstable manifolds are well defined.

- Assume that $y \in \Gamma_{\varepsilon}(x) \cap W_{\text {loc }}^{\text {cu }}(x)$. Then the center-unstable arc $[x, y]^{\text {cu }} \subset$ $W_{\mathrm{loc}}^{\mathrm{cu}}(x)$ is a $(\varepsilon, E)$-interval [33], and therefore by the domination property (1), $W_{\text {loc }}^{\mathrm{cs}}(z)$ is a true stable manifold for all $z \in[x, y]^{\mathrm{cu}}$, that is, $W_{\mathrm{loc}}^{\mathrm{cs}}\left([x, y]^{\mathrm{cu}}\right)$ contains a neighborhood in $M$.

- By [33, Proposition 3.1], either $\ell\left(f^{n}\left([x, y]^{\mathrm{cu}}\right)\right) \rightarrow 0$ when $n \rightarrow+\infty$ or the $\omega$-limit set of $[x, y]^{\mathrm{cu}}$ is contained in a periodic arc or a periodic circle.

(i) In the first case $f^{n}\left(W_{\text {loc }}^{\text {cs }}\left([x, y]^{\text {cu }}\right)\right)$ shrinks to a point when $n \rightarrow+\infty$. This implies that the topological entropy of $W_{\mathrm{loc}}^{\mathrm{cs}}\left([x, y]^{\mathrm{cu}}\right)$ vanishes and therefore we also have $h\left(W_{\text {loc }}^{\text {cs }}\left([x, y]^{\text {cu }}\right) \cap \Gamma_{\varepsilon}(x)\right)=0$.

(ii) If the $\omega$-limit set of $W_{\mathrm{loc}}^{\mathrm{cs}}\left([x, y]^{\mathrm{cu}}\right)$ is contained in a periodic arc or a periodic circle then there is $N$ such that for $n>N$ the dynamics on $f^{n}\left(W_{\text {loc }}^{\text {cs }}\left([x, y]^{\text {cu }}\right)\right)$ is, for $n$ large enough, similar to the dynamics of the diffeomorphism restricted to that interval or circle which always has zero topological entropy. This implies again that $h\left(W_{\mathrm{loc}}^{\mathrm{cs}}\left([x, y]^{\mathrm{cu}}\right)\right)=0$ too.

In any case we derive that $h\left(W_{\mathrm{loc}}^{\mathrm{cs}}\left([x, y]^{\mathrm{cu}}\right), f\right)=0$, implying that $h\left(W_{\mathrm{loc}}^{\mathrm{cs}}\left([x, y]^{\mathrm{cu}}\right) \cap \Gamma_{\varepsilon}(x)\right)=0$. Similar arguments are valid for $y \in W_{\mathrm{loc}}^{\mathrm{cs}}(x)$. In this case we have that $[x, y]^{\text {cs }} \subset W_{\text {loc }}^{\text {cs }}(x)$ is a $(\varepsilon, F)$-interval.

- If $y \in \Gamma_{\varepsilon}(x)$ is such that $y \notin W_{\mathrm{loc}}^{\mathrm{cu}}(x) \cup W_{\mathrm{loc}}^{\mathrm{cs}}(x)$ then we project $y$ along $W_{\mathrm{loc}}^{\mathrm{cs}}(y)$ into $W_{\mathrm{loc}}^{\mathrm{cu}}(x)$ obtaining a point $y_{F}$ that is contained in $\Gamma_{L \cdot \varepsilon}(x)$ for some constant $L>0$ independent of $y$, and argue as above. The existence of $L$ is due to the fact that the angle between $E(z)$ and $F(z)$ is bounded away from zero for any point $z \in U(K)$.

- So in any case we conclude that $\Gamma_{\varepsilon}(x)$ is contained in the local stable manifold of some $(L \cdot \varepsilon, E)$-interval or in the local unstable manifold of some $(L \cdot \varepsilon, F)$ interval and therefore $h\left(\Gamma_{\varepsilon}(x), f\right)=0$. Since this last equality holds for all $x \in K$, Theorem A follows.

To prove Theorem B we proceed as follows.

- We use the existence of the finest dominated splitting (see Definition 3.1) for a homoclinic class $H(p, f)$. Generically, in the $C^{1}$ topology, this splitting has the form

$$
T_{H(p, f)} M=E \oplus F_{1} \oplus \cdots \oplus F_{j-i} \oplus G
$$


with $\operatorname{dim}(E)=i, \operatorname{dim}\left(F_{h}\right)=1$ for all $h$, and $\operatorname{dim}(G)=\operatorname{dim}(M)-j$. The sub-bundles $F_{h}$ are not hyperbolic $[1,19]$.

- When $H(p, f)$ is an isolated homoclinic class it is known that $E$ is uniformly contracting and $G$ is uniformly expanding [4].

- Take $\varepsilon>0$ such that the dominated splitting extends to any point whose orbit is at a distance less than $\varepsilon$ from the orbit of a point in $H(p, f)$. Thus if for some $x \in H(p, f)$ there is $y \in \Gamma_{\varepsilon}(x)$ then $y$ cannot be in the strong unstable manifold of $x$ (tangent to $G$ ) neither in the strong stable manifold (tangent to $E$ ).

(i) $y$ cannot project along its center-stable manifold into the unstable manifold of $x$ into $y_{G} \neq x$. Otherwise, taking into account that the angles between any distinct sub-bundle is bounded away from zero we get that $\forall n \in \mathbb{Z}$ : $f^{n}\left(y_{G}\right) \in \Gamma_{\varepsilon \cdot L}\left(f^{n}(x)\right)$ where $L>0$ is some constant. Forward iterations of the distance between $f^{n}(x)$ and $f^{n}\left(y_{G}\right)$ growths exponentially fast until $f^{n}\left(y_{G}\right)$ leaves $W_{\text {loc }}^{u}\left(f^{n}(x)\right)$. Hence $y_{G} \neq x$ leads to a contradiction with the fact that $y \in \Gamma_{\varepsilon}(x)$.

(ii) Thus $y$ lies in a center manifold. Since the tangent bundle to this center manifold splits into one-dimensional ones, repeating the arguments used in Theorem A we get the proof of Theorem B.

To prove the remaining theorem we introduce the notions of symbolic extension and principal symbolic extension. Let $f: X \rightarrow X$ be a homeomorphism of the compact metric space $X$. A symbolic extension of $(f, X)$ is a subshift on a finite alphabet $(g, Y)$ which has $f$ as a topological factor. $(g, Y)$ is an extension of $(f, X)$ if there exists a continuous surjection $\pi: Y \rightarrow X$ such that $f \pi=\pi g$.

For any $f$-invariant measure $\mu$ we define $h_{\text {ext }}^{\pi}(\mu)=\sup \left\{h_{\nu}(g): \pi_{*} \nu=\mu\right\}$, where $h_{\nu}(g)$ is the entropy of $g$ with respect to $\nu$. An extension $(g, Y)$ is principal if it preserves the measure theoretic entropy of the factor $(f, X)$ for any $f$-invariant measure $\mu$, i.e., $h_{\mathrm{ext}}^{\pi}(\mu)=h_{\mu}(f)$. Roughly speaking principal extensions preserves the entire information theory of the original system.

The proof of Theorem $\mathrm{C}$ is obtained following the steps:

- We profit from a result of Downarowicz and Newhouse, [15, Theorem 1.4], that shows that if we have a Hènon like tangency between the stable manifold and the unstable manifold of a periodic point then $f$ cannot have a principal symbolic extension.

- Under the assumption that we do not have a dominated splitting but that we have robust entropy expansiveness, we can perturb $f$ to create a tangency between the stable manifold and the unstable manifold of the continuation $p_{g}$ of the $f$-periodic point $p$. After a new perturbation we may assume that this tangency is a Hènon like one and that the perturbed diffeomorphism is $C^{2}$. 
- Applying the results of [15] we obtain that $f / H\left(p_{g}, g\right)$ cannot have a principal symbolic extension.

- On the other hand it is proved in [6] that for a homeomorphism defined on a compact metric space it is equivalent to have a principal symbolic extension and to be asymptotically entropy expansive.

- Joining the last two facts we arrive to a contradiction since an entropy expansive system is asymptotically entropy expansive.

\section{Examples}

Observe that since for Morse-Smale diffeomorphisms topological entropy vanishes they are a fortiori $h$-expansive. Moreover, they are robust under $C^{1}$ perturbations, so we cannot expect in general to have a dominated splitting when we have robust entropy-expansiveness. That is the reason for which we restrict ourselves to the case of homoclinic classes in Theorem C.

In [7] Bowen asked for examples of diffeomorphisms which are not $h$-expansive. The first giving such an example was Misiurewicz, [26]. Nevertheless we give here a $C^{\infty}$ example in $S$ which illustrates the fact that we should have "arbitrarily small" horseshoes to brake $h$-expansiveness. By "arbitrarily small horseshoe" we mean a horseshoe contained in the intersection of the $\varepsilon$-stable and unstable manifolds for any positive $\varepsilon$. A modification of such example gives a 3-dimensional one which does have a dominated splitting but is not $h$-expansive illustrating that in the general case dominance is not enough to guarantee $h$-expansiveness. As Theorem B shows, the existence of a dominated splitting implies $h$-expansiveness generically in the case of isolated homoclinic classes.

Example 1.1. There is a $C^{\infty}$ diffeomorphism of $S^{2}$ that is not h-expansive.

We consider in $\mathbb{R}^{2}$ the action given by the matrix $A=\left(\begin{array}{ll}2 & 1 \\ 1 & 1\end{array}\right)$. Since the entries of $A$ are integers and $\operatorname{det}(A)=1$, the lattice $\mathbb{Z}^{2}$ is preserved by this action and therefore it passes to the quotient $\mathbb{T}^{2}=\mathbb{R}^{2} / \mathbb{Z}^{2}$. This gives us a very well known linear Anosov diffeomorphism $a: \mathbb{T}^{2} \rightarrow \mathbb{T}^{2}$. Let $[x, y]$ represent the equivalence class of $(x, y) \in \mathbb{R}^{2}$ in $\mathbb{T}^{2}$. We define in $\mathbb{T}^{2}$ the relation $[x, y] \sim[-x,-y]=-[x, y]$. The quotient $\mathbb{T}^{2} / \sim$ gives the sphere $S^{2}$. In order to see this let us take the square in $\mathbb{R}^{2}$ limited by the straight lines $x=-\frac{1}{2}, x=\frac{1}{2}, y=-\frac{1}{2}, y=\frac{1}{2}$. We obtain a fundamental domain for the torus and we identify it with $\mathbb{T}^{2}$.

In the quotient $\mathbb{T}^{2}$ the vertices $\mathrm{A}(1 / 2,1 / 2), \mathrm{B}(-1 / 2,1 / 2), \mathrm{C}(-1 / 2,-1 / 2)$, $\mathrm{D}(1 / 2,-1 / 2)$, of the square are all identified. Let us call $\mathrm{E}$ to the point $(1 / 2,0)$, $\mathrm{F}$ to the point $(-1 / 2,0), \mathrm{G}$ to the point $(0,1 / 2)$, and $\mathrm{H}$ to the point $(0,-1 / 2)$. Observe that $\mathrm{E}$ is identified with $\mathrm{F}$ and $\mathrm{G}$ is identified with $\mathrm{H}$ in $\mathbb{T}^{2}$. Now observe that the boundary of the square OEAG is identified with the boundary of the square OEDH (by the relations $(x, y) \sim-(x, y)$ and $(x, y) \sim\left(x^{\prime}, y^{\prime}\right)$ if $\left.\left(x-x^{\prime}, y-y^{\prime}\right) \in \mathbb{Z}^{2}\right)$. Hence 


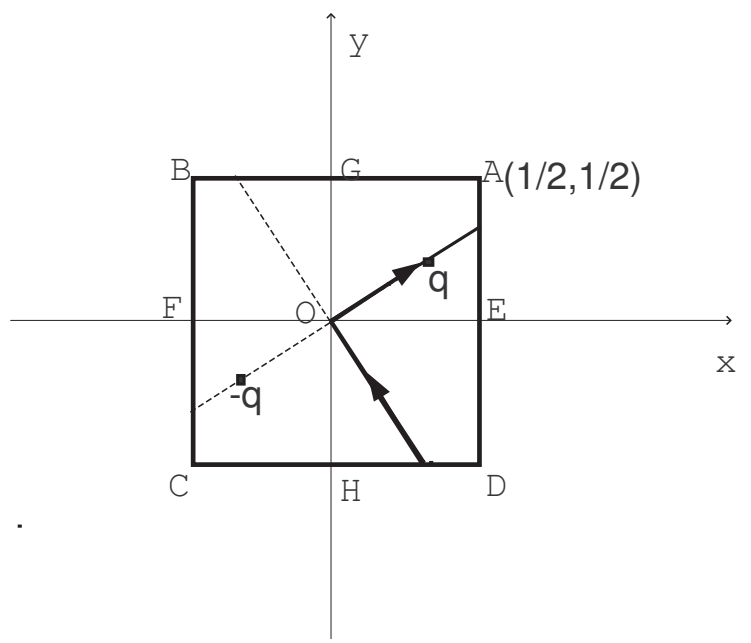

Figure 1 - Fundamental domain for $\mathbb{T}^{2}$

both squares are two different disks glued in their boundaries by this identification. It is not difficult to see that the quotient topology coincides in the interior of the squares OEAG and OEDH with the topology of $\mathbb{R}^{2}$ and that the common boundary of both disks is a circle separating $\mathbb{T}^{2} / \sim$ Moreover, the rest of the square ABCD doesn't add more points to the quotient because the squares OEAG and OFCH, and $\mathrm{OEDH}$ and OFBG, are identified by the relation $(x, y) \sim-(x, y)$. Hence we obtain that $\mathbb{T}^{2} / \sim \cong S^{2}$. See figure 1 where we have marked two points, $q$ and $-q$, which are identified by the relation $\sim$.

On the other hand $a([x, y]) \sim-a([x, y])=a(-[x, y])$ by linearity, and therefore projects to $S^{2}$ as a map $g: S^{2} \rightarrow S^{2}$, known as a generalized pseudo-Anosov map which is shown to be Bernoulli with respect to Lebesgue [17]. If $\Pi: \mathbb{T}^{2} \rightarrow S^{2}$ is the projection defined by the relation $\sim$, we may write $g(x)=\Pi\left(a\left(\Pi^{-1}(x)\right)\right)$. Observe that the projection $\Pi: \mathbb{T}^{2} \rightarrow S^{2}$ is a branched covering and that the definition of $g$ doesn't depend on the pre-image of $x$ by $\Pi^{-1}$. Therefore periodic points of $a$ project into periodic points of $g$ and dense orbits of $a$ project into dense orbits of $g$. For $g$ there are singular points $P$ where the local $\varepsilon$-stable and $\varepsilon$-unstable sets are arcs with the point $P$ as an end-point. This local stable (unstable) sets are called 1-prongs (see figures 1 and 2 where $O$ is a point with 1-prongs).

Let $O \in S^{2}$ be the image by $\Pi$ of $[0,0]$. Then $O$ is a fixed point of $g$. The point $O$ is singular because the local stable and unstable manifolds of $[0,0]$ in $\mathbb{T}^{2}$ project into $S^{2}$ as arcs ending at $O$ (because $[x, y] \sim-[x, y]$ ). The local stable and unstable manifolds of the points in $\mathbb{T}^{2}$ near $[0,0]$ project onto arcs contained in the stable and unstable sets respectively of points in $S^{2}$ near $O$ like in figure 2. Note that we do not speak of 


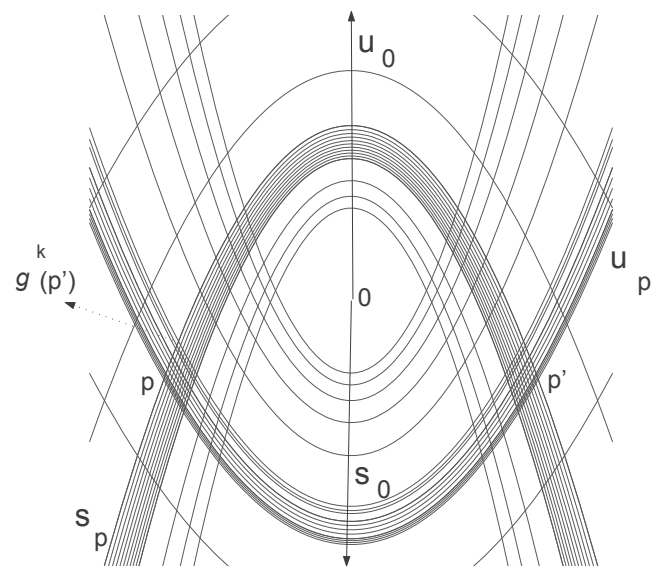

Figure 2 - Singularity of a generalized pseudo-Anosov

stable (unstable) manifolds but of stable (unstable) sets because neither $W_{\text {loc }}^{\mathrm{s}}(x)$ nor $W_{\text {loc }}^{\mathrm{u}}(x)$ are locally connected (see [30]).

The intersection of the stable and unstable manifolds of the points $[0, x]$ and $[0,-x]$ of $\mathbb{T}^{2}$ consists of four points identified by pairs by the relation $[x, y] \sim-[x, y]$. If $[x, y] \in \mathbb{T}^{2}$ projects to $X \in S^{2}$, let us call $s_{X}$ and $u_{X}$ to the projections of the $\varepsilon$-local stable and $\varepsilon$-local unstable manifolds respectively of the point $[x, y]$. Hence if a point $X$ is close enough to a singular point like $O, s_{X}$ and $u_{X}$ will intersect twice. Points in $s_{X}$ are in the $\varepsilon$-local stable set of $X$ and points in $u_{X}$ are in the $\varepsilon$-local unstable set of $X$. Moreover, if $Y \in s_{X}$ then $\operatorname{dist}\left(g^{n}(Y), g^{n}(X)\right) \rightarrow 0$ when $n \rightarrow+\infty$. Similarly for points in $u_{X}$ replacing $n \rightarrow+\infty$ by $n \rightarrow-\infty$.

Given $\varepsilon^{\prime}>0$ choose a periodic point $p \in \mathbb{T}^{2}$ so close to $[0,0]$ that $\Pi(p)=P$ is a periodic point satisfying $\operatorname{dist}(P, 0)<\varepsilon^{\prime}$. Such a point exists since periodic points are dense for the Anosov diffeomorphism $a$ defined on $\mathbb{T}^{2}$ and projects on $S^{2}$ as $g$-periodic points.

Let $\left\{P, P^{\prime}\right\}=s_{P} \cap u_{P}$. Then it is not difficult to see that given $\varepsilon>0$ there is $\varepsilon^{\prime}>0$ small enough such that $P^{\prime} \in W_{\varepsilon}^{u}(P) \cap W_{\varepsilon}^{s}(P)$. Thus we have a homoclinic intersection between $\varepsilon$-local stable and $\varepsilon$-local unstable arcs of the periodic point $P$, where $P^{\prime}$ is a homoclinic point associated to $P$.

It follows that for all $\varepsilon>0$ there are points $P$ such that $\Gamma_{\varepsilon}(P)$ contains a small horseshoe. Thus $g: S^{2} \rightarrow S^{2}$ is not $h$-expansive since the topological entropy of horseshoes is positive. Moreover, this example is transitive and there are analytic models for it (see [17], and [22]).

Clearly the example is a homoclinic class which has no dominated splitting.

Example 1.2. Property (1) sole does not imply h-expansiveness in dimension 3 or more. 
Consider the 3-manifold $M=S^{2} \times S^{1}$ with $g: S^{2} \rightarrow S^{2}$ as in the example above, and put in $S^{1}$ a diffeomorphism $h: S^{1} \rightarrow S^{1}$ with a North-South dynamics, say, $N \in S^{1}$ is a source and $S \in S^{1}$ is a sink and the $\omega$-limit of any point in $S^{1}$ is $S$ and the $\alpha$-limit of every point in $S^{1}$ is $N$. We may assume that $\left|D h_{N}\right|>2 k$ where $k=\sup \left\{\|D g(x)\|, x \in S^{2}\right\}$. Let us define $f: M \rightarrow M$ by $f(x, y)=(g(x), h(y))$. Then if $K=S^{2} \times\{N\} \subset M, K$ is compact invariant and there is a dominated splitting for $K, E \oplus F$, where $E=T_{x} S^{2}, F=T_{N} S^{1}$. By the previous example $f$ is not $h$-expansive.

This example shows what may occur to prevent a diffeomorphism $f$ defined on an invariant set $K$ supporting a dominated splitting to be $h$-expansive: the strongly expanding direction $F$ along $T_{S^{1}} M$ does not interferes on the dynamics of $f / S^{2} \times\{N\}$. Thus property (1) holds for $f$ restricted to $K=S^{2} \times\{N\}$ albeit does not for the projection $g=\Pi_{S^{2}} f$. In fact $K$ is a (flat) homoclinic class for $f: M \rightarrow M$.

\section{Dominated splitting implies $h$-expansiveness on surfaces}

Here we shall prove Theorem A. Let us begin stating the following lemma.

Lemma 2.1 (Pliss). Let $0<\lambda_{1}<\lambda_{2}<1$ and assume that there exists $n>0$ arbitrarily large such that

$$
\prod_{j=1}^{n}\left\|D f / E\left(f^{j}(x)\right)\right\| \leq \lambda_{1}^{n} .
$$

Then there exist a positive integer $N=N\left(\lambda_{1}, \lambda_{2}, f\right), c=c\left(\lambda_{1}, \lambda_{2}, f\right)>0$, such that if $n \geq N$ then there exist numbers

$$
0 \leq n_{1} \leq n_{2} \leq \cdots \leq n_{l} \leq n
$$

such that

$$
\prod_{j=n_{r}}^{h}\left\|D f / E\left(f^{j}(x)\right)\right\| \leq \lambda_{2}^{h-n_{r}},
$$

for all $r=1,2, \ldots, l$, with $l \geq c n$, and for all $h$ with $n_{r} \leq h \leq n$.

Proof. See [31].

Let $M$ be a surface and $K \subset M$ a compact and $f$-invariant subset such that $T_{K}(M)$ can be written as a dominated splitting $E \oplus F$ verifying (1). By continuity of $f$ and $D f$ there is $\delta_{0}>0$ such that we may extend the cones defining the dominated splitting to the closed $\delta_{0}$-neighborhood of $K, U(K)=\left\{y \in M \mid \operatorname{dist}(y, K) \leq \delta_{0}\right\}$. In this neighborhood there exists a continuous splitting $T_{U(K)}(M)=\hat{E} \oplus \hat{F}$ extending the splitting $T_{K}(M)=E \oplus F$ (see [24]). If the orbit of a point $y$, orb $(y)$, is contained in $U(K)$ then for that point there are defined local center-stable and center-unstable 
manifolds $W_{\text {loc }}^{\text {cs }}(y)$ and $W_{\text {loc }}^{\text {cu }}(y)$ where loc $>0$ stands for a small real number. By Peano Theorem on the existence of solutions of an ordinary differential equation given by a continuous field, $W_{\mathrm{loc}}^{\text {cs }}(y)$ can be obtained as a solution of the ODE

$$
\left\{\begin{array}{l}
Y(0)=y \\
Y^{\prime}(u)=\hat{E}(u), \quad u \in \mathbb{R}^{2}
\end{array}\right.
$$

where we have identified $B\left(y, \delta_{0}\right)$ with $\mathbb{R}^{2}$. Such a solution is tangent to $\hat{E}$ and therefore, by domination, it is a local center-stable manifold for $y$. Similarly for $\hat{F}$ we obtain $W_{\text {loc }}^{\text {cu }}(y)$ as a solution of the ODE obtained replacing $\hat{E}$ by $\hat{F}$. We may also assume that for all $x \in M$ the $\delta_{0}$-neighborhood of $x, B\left(x, \delta_{0}\right)$, is contained in a local chart and therefore we can identify $B\left(x, \delta_{0}\right)$ with $\mathbb{R}^{2}$. Moreover, there is $\delta_{1}, 0<\delta_{1} \leq \delta_{0}$, such that if $\operatorname{dist}\left(f^{j}(y), f^{j}(z)\right) \leq \delta_{1}$ for all $j=0, \ldots, n$ and $z \in W_{\text {loc }}^{\text {cs }}(y)$ then $f^{j}(z) \in W_{\text {loc }}^{\text {cs }}\left(f^{j}(y)\right)$ for all $j=0, \ldots, n$. Similarly for the local center-unstable manifolds we have that if $\operatorname{dist}\left(f^{-j}(y), f^{-j}(z)\right) \leq \delta_{1}$ for all $j=0, \ldots, n$ and $z \in W_{\mathrm{loc}}^{\mathrm{cs}}(y)$ then $f^{-j}(z) \in W_{\mathrm{loc}}^{\mathrm{cs}}\left(f^{-j}(y)\right)$ for all $j=0, \ldots, n$ (see [33, Lemma 3.0.4 and Corollary 3.2]).

Let us denote by $\hat{K}$ the maximal $f$-invariant subset of $U(K)$ and by $\hat{K}^{+}$and $\hat{K}^{-}$ the forward and backward maximal invariant subsets respectively:

$$
\hat{K}=\bigcap_{j \in \mathbb{Z}} f^{j}(U(K)), \quad \hat{K}^{+}=\bigcap_{j=0}^{\infty} f^{-j}(U(K)), \quad \hat{K}^{-}=\bigcap_{j=0}^{\infty} f^{j}(U(K)),
$$

The following lemma relates the length of a stable (unstable) arc joining two points with the distance between those points.

Lemma 2.2. Given $y \in \hat{K}^{+}$there is $\delta_{2}, 0<\delta_{2} \leq \delta_{1}$, such that if the length of the arc $[y, z]^{\mathrm{cs}} \subset W_{\mathrm{loc}}^{\mathrm{cs}}(y)$ is greater than $\delta>0$ for $0<\delta \leq \delta_{2}, \ell\left([y, z]^{\mathrm{cs}}\right)>\delta$, then $\operatorname{dist}(y, z)>\delta / 2$. Moreover, we may choose $\delta_{2}$ such that if $\operatorname{dist}(y, z) \leq \delta \leq \delta_{2}$ then $\ell\left([y, z]^{\mathrm{cs}}\right) \leq 2 \cdot \delta$. Similarly for an arc $[y, z]^{\mathrm{cu}} \subset W_{\mathrm{loc}}^{\mathrm{cu}}(y), y \in \hat{K}^{-}$.

Proof. Since $\hat{E}$ is a continuous sub-bundle of $T_{U(K)} M$ we may find $\delta_{2}, 0<\delta_{2} \leq \delta_{1}$ such that given $\pi / 8 \geq \eta>0$ then the angle $\angle(E(y), E(w))<\eta$ for all $w \in B\left(y, \delta_{2}\right) \cap U(K)$. Thus if we parameterize $[y, z]^{\text {cs }}$ by arc-length $\beta:[0, l] \rightarrow M, \beta(s)=\left(\beta_{1}(s), \beta_{2}(s)\right)$, with $\beta(0)=y, \beta(l)=z$, then $\beta^{\prime}(s)=\left(\beta_{1}^{\prime}(s), \beta_{2}^{\prime}(s)\right)$ is parallel to $E(\beta(s))$, here we have put $l=\operatorname{length}\left([y, z]^{\mathrm{cs}}\right)$. Therefore, since $\left(\beta_{1}^{\prime}(s)\right)^{2}+\left(\beta_{2}^{\prime}(s)\right)^{2}=1$, we have by the 


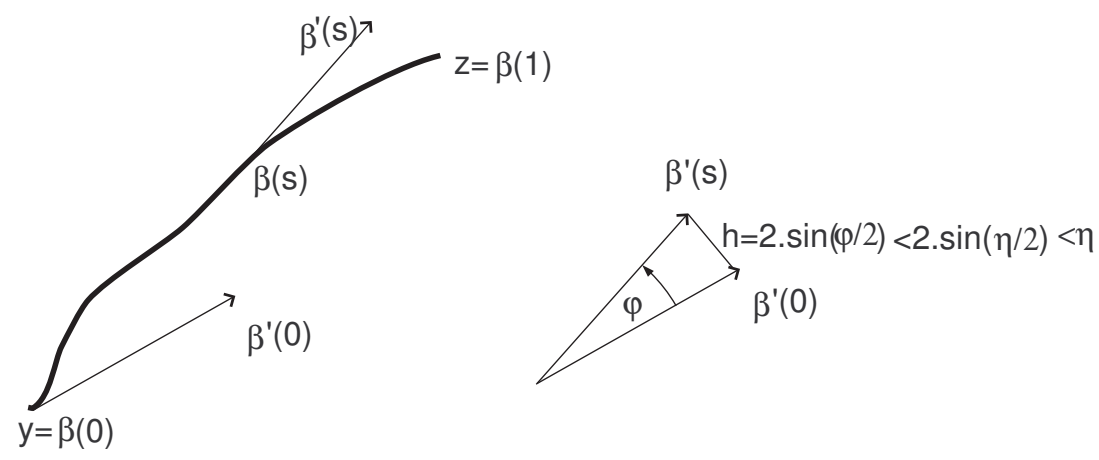

Figure 3 - Bounds for small angles

Mean Value Theorem

$$
\begin{aligned}
\operatorname{dist}(y, z)= & \|\beta(l)-\beta(0)\|=\sqrt{\left(\beta_{1}(l)-\beta_{1}(0)\right)^{2}+\left(\beta_{2}(l)-\beta_{2}(0)\right)^{2}} \\
= & \sqrt{\left(\beta_{1}^{\prime}\left(s_{1}\right)\right)^{2}+\left(\beta_{2}^{\prime}\left(s_{2}\right)\right)^{2}} \cdot l \\
= & l\left(1-\left(\sqrt{\left(\beta_{1}^{\prime}(0)\right)^{2}+\left(\beta_{2}^{\prime}(0)\right)^{2}}-\sqrt{\left.\left(\beta_{1}^{\prime}\left(s_{1}\right)\right)^{2}+\left(\beta_{2}^{\prime}\left(s_{2}\right)\right)^{2}\right)}\right)\right. \\
= & l\left(1-\frac{\left(\beta_{1}^{\prime}(0)\right)^{2}-\left(\beta_{1}^{\prime}\left(s_{1}\right)\right)^{2}+\left(\beta_{2}^{\prime}(0)\right)^{2}-\left(\beta_{2}^{\prime}\left(s_{2}\right)\right)^{2}}{1+\sqrt{\left(\beta_{1}^{\prime}\left(s_{1}\right)\right)^{2}+\left(\beta_{2}^{\prime}\left(s_{2}\right)\right)^{2}}}\right) \\
\geq & l\left(1-\left|\beta_{1}^{\prime}(0)-\beta_{1}^{\prime}\left(s_{1}\right)\right|\left|\beta_{1}^{\prime}(0)+\beta_{1}^{\prime}\left(s_{1}\right)\right|\right. \\
& \left.+\left|\beta_{2}^{\prime}(0)-\beta_{2}^{\prime}\left(s_{2}\right)\right|\left|\beta_{2}^{\prime}(0)+\beta_{2}^{\prime}\left(s_{2}\right)\right|\right) .
\end{aligned}
$$

But, since $\angle(\hat{E}(\beta(s)), \hat{E}(\beta(0)))<\eta$,

$$
\left\|\left(\beta_{1}^{\prime}(s)-\beta_{1}^{\prime}(0), \beta_{2}^{\prime}(s)-\beta_{2}^{\prime}(0)\right)\right\| \leq 2 \sin (\eta / 2)<\eta,
$$

(see figure 3). Therefore, taking into account that

$$
\left|\beta_{1}^{\prime}(0)+\beta_{1}^{\prime}\left(s_{1}\right)\right| \leq\left|\beta_{1}^{\prime}(0)\right|+\left|\beta_{1}^{\prime}\left(s_{1}\right)\right| \leq 2
$$

and that the same is true with respect to $\beta_{2}^{\prime}$ we have

$$
\operatorname{dist}(y, z) \geq l(1-4 \eta)>l / 2>\delta / 2
$$

if $\eta>0$ is sufficiently small. 
To prove that if $\operatorname{dist}(y, z) \leq \delta$ then $\ell\left([y, z]^{\text {cs }}\right) \leq 2 \delta$ let us assume that $y$ is the origin $O$ of coordinates in $\mathbb{R}^{2}$ and that the $O x_{1}$ axis is in the direction of $\hat{E}(y)$. Since $\angle(\hat{E}(y), \hat{E}(w))<\eta \leq \pi / 8$ for all $w \in B\left(y, \delta_{2}\right)$, all the solutions starting at $y$ are contained in the cone of center $y=O$, axis $O x_{1}$ and angle with $O x_{1}$ equal to $\eta$. It follows that the arc $[y, z]^{\mathrm{cs}}$ of the local center stable manifold of $y$ is contained in that cone and that the local center stable manifold of $y$ can be written as the graph of a $C^{1}$ function $x_{2}=h\left(x_{1}\right)$. Moreover $\left|h^{\prime}\left(x_{1}\right)\right| \leq \tan (2 \eta) \leq 4 \eta$, whenever $0 \leq \eta \leq \pi / 8$. Moreover, since $\eta \leq \pi / 8$ we have $\sqrt{1+16 \eta^{2}}<\sqrt{1+16 \times(0.4)^{2}}<2$. Thus, if $x_{1}(z)$ denotes the abscissa of $z$, since $\left|x_{1}(z)\right| \leq \operatorname{dist}(y, z) \leq \delta$, then

$$
\begin{aligned}
\ell\left([y, z]^{\mathrm{cs}}\right) & =\int_{0}^{x_{1}(z)} \sqrt{1+h^{\prime 2}\left(x_{1}\right)} d x_{1} \\
& \leq \int_{0}^{\delta} \sqrt{1+16 \eta^{2}} d x_{1}=\delta \sqrt{1+16 \eta^{2}} \leq 2 \cdot \delta .
\end{aligned}
$$

Proof of Theorem A. We first observe that taking an iterate $f^{m}$ of $f$ we may assume $C=1$ at equation (1) defining domination in order to simplify calculations. Indeed, we have for all $n \geq 1$

$$
\begin{aligned}
\left\|D\left(f^{m}\right)^{n} / E(x)\right\| \cdot\left\|D\left(f^{m}\right)^{-n} / F\left(f^{m n}(x)\right)\right\| & =\left\|D f^{m n} / E(x)\right\| \cdot\left\|D f^{-m n} / F\left(f^{m n}(x)\right)\right\| \\
& \leq C \lambda^{m n}=C\left(\lambda^{m}\right)^{n} \leq \lambda^{\prime n},
\end{aligned}
$$

if we choose $1>\lambda^{\prime}>\lambda$ and $m>0$ such that $C \leq \lambda^{\prime} / \lambda^{m}$. Since for a compact invariant set $X$ we have that the topological entropy $h\left(f^{m} / X\right)=m \cdot h(f / X)$, if we prove that for some $\varepsilon>0, h\left(f^{m} / \Gamma_{\varepsilon}(x, f)\right)=0$ then the same is true for $f$. Thus we assume that for $f$ itself $C=1$ and $\lambda=\lambda^{\prime}$. Let $\sqrt{\lambda}<\lambda_{1}<\lambda_{2}<\lambda_{3}<1$. We find $\delta_{3}$, $0<\delta_{3} \leq \delta_{2}$, such that if $\operatorname{dist}(z, w) \leq \delta_{3}, z, w \in U(K)$, then

$$
1-c<\frac{\|D f / \hat{E}(z)\|}{\|D f / \hat{E}(w)\|}<1+c \quad \text { and } \quad 1-c<\frac{\left\|D f^{-1} / \hat{F}(z)\right\|}{\left\|D f^{-1} / \hat{F}(w)\right\|}<1+c,
$$

where $c>0$ is such that $(1+c) \lambda_{2} \leq \lambda_{3}$.

Since $U(K)$ is a compact neighborhood of $K$ and $T_{U(K)} M=\hat{E} \oplus \hat{F}$ is a dominated splitting we may find $\gamma>0$ such that for all $y \in U(K)$ it holds $\angle(\hat{E}(y), \hat{F}(y)) \geq \gamma$. Let us pick a point $x \in K$ and, identifying $\mathbb{R}^{2}$ with a coordinate neighborhood around $x$, let $l_{E}(x)$ be the straight line at $x$ tangent to $E(x)$ and $l_{F}(x)$ the straight line tangent to $F(x)$. From a point $y_{F} \in l_{F}(x), y_{F} \neq x$, we consider the straight line $y_{F}+l_{E}(x)$ parallel to $E(x)$. Then for any point $y$ in $y_{F}+l_{E}(x)$ we have that the distance between $y$ and $x$ is greater than the distance between $y_{F}$ and $x$ multiplied by $\sin \gamma$, that is,

$$
\operatorname{dist}(y, x) \geq \operatorname{dist}\left(y_{F}, x\right) \sin \gamma \Longrightarrow \operatorname{dist}\left(y_{F}, x\right) \leq \frac{\operatorname{dist}(y, x)}{\sin \gamma},
$$

(see figure 4). 


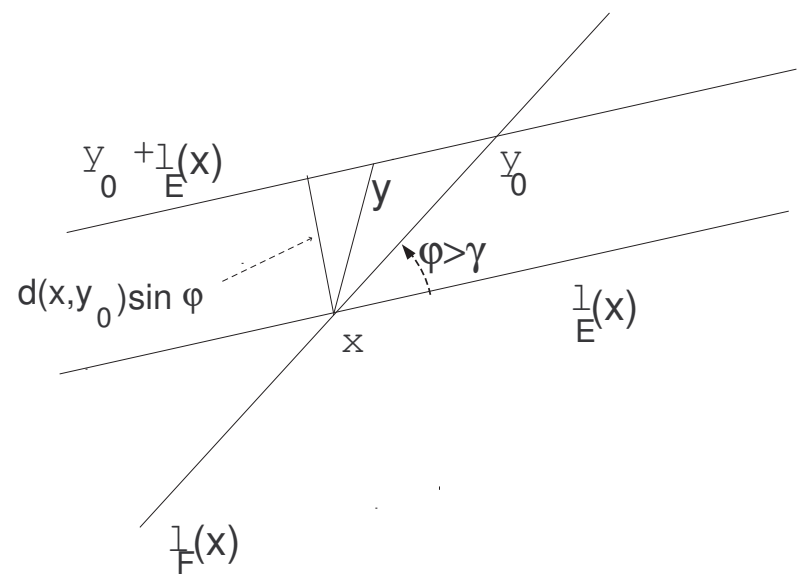

Figure 4 - Bounds for the distance between $x$ and $y \in y_{0}+l_{E}(x)$

When we substitute the linear model by that given by the local center-stable and center-unstable manifolds, since the local center-unstable manifold is tangent to $\hat{F}$ and the local center-stable manifold is tangent to $\hat{E}$ we may assume that $\delta_{3}$ is so small that

$$
\operatorname{dist}(y, x) \geq \operatorname{dist}\left(y_{F}, x\right)\left(\frac{\sin \gamma}{3}\right) \Longrightarrow \operatorname{dist}\left(y_{F}, x\right) \leq \frac{3 \operatorname{dist}(y, x)}{\sin \gamma}
$$

for $y_{F} \in W_{\mathrm{loc}}^{\mathrm{cu}}(x) \cap B\left(x, \delta_{3}\right), y \in W_{\mathrm{loc}}^{\mathrm{cs}}\left(y_{F}\right) \cap B\left(x, \delta_{3}\right)$.

Now let $\varepsilon>0$ be such that

$$
\varepsilon<\frac{\delta_{3} \sin \gamma}{6}
$$

We will prove that for all $x \in K, h\left(f / \Gamma_{\varepsilon}(x)\right)=0$, thus proving that $f / K$ is $h$ expansive.

Let us first assume that $y \in W_{\mathrm{loc}}^{\mathrm{cu}}(x) \cap \Gamma_{\varepsilon}(x), y \neq x$, and in order to simplify notation let us put $E(F)$ instead of $\hat{E}(\hat{F})$ in the sequel. Since $y \in \Gamma_{\varepsilon}(x)$ we have that $\operatorname{orb}(y) \subset U(K)$ and so $y \in \hat{K}$. Therefore for all $j \in \mathbb{Z}$ it holds that

$$
\left\|D f / E\left(f^{j-1}(y)\right)\right\| \cdot\left\|D f^{-1} / F\left(f^{j}(y)\right)\right\|<\lambda
$$

and so

$$
\prod_{j=1}^{n}\left\|D f / E\left(f^{j-1}(y)\right)\right\| \cdot\left\|D f^{-1} / F\left(f^{j}(y)\right)\right\|<\lambda^{n}, \forall n \geq 1 .
$$


If it were the case that

$$
\prod_{j=1}^{n}\left\|D f^{-1} / F\left(f^{j}(y)\right)\right\| \leq \lambda_{1}^{n}
$$

for arbitrarily large $n>0$ then by Lemma 2.1 there are $N=N\left(\lambda_{1}, \lambda_{2}\right) \in \mathbb{N}$ and $c=c\left(\lambda_{1}, \lambda_{2}\right)>0$ such that if $n \geq N$ there exists $1 \leq n_{1}<n_{2}<\cdots<n_{k} \leq n$ with $k>c \cdot n$ and

$$
\prod_{j=h}^{n_{i}}\left\|D f^{-1} / F\left(f^{j}(y)\right)\right\| \leq \lambda_{2}^{n_{i}-h},
$$

for $n_{i} \geq h \geq 1, i=1, \ldots, k$. In particular $n_{k} \geq k>c \cdot n$. Thus by our choice of $\delta_{3}$ and $\varepsilon$ we have that

$$
\prod_{j=h}^{n_{k}}\left\|D f^{-1} / F\left(f^{j}(z)\right)\right\| \leq \lambda_{3}^{n_{1}-h},
$$

for all $h\left(n_{k} \geq h \geq 1\right)$ if $\operatorname{dist}\left(f^{j}(z), f^{j}(y)\right) \leq \delta_{3}$ for all $j\left(h \leq j \leq n_{k}\right)$ and $f^{j}(y) \in W_{\mathrm{loc}}^{\mathrm{cu}}\left(f^{j}(x)\right)$ for all $j \geq 0$. Moreover $f^{j}\left([x, y]^{\mathrm{cu}}\right) \subset W_{\mathrm{loc}}^{\mathrm{cu}}\left(f^{j}(x)\right)$.

If $\rho=\operatorname{dist}(x, y)>0$, we have for $h=1$

$$
\rho \leq \ell\left([x, y]^{\mathrm{cu}}\right) \leq \ell\left(\left[f^{n_{k}}(x), f^{n_{k}}(y)\right]^{\mathrm{cu}}\right) \lambda_{3}^{n_{k}-1} .
$$

Since $\left[f^{n_{k}}(x), f^{n_{k}}(y)\right]^{\text {cu }}$ is tangent to $F$ and $\operatorname{dist}\left(f^{n_{k}}(x), f^{n_{k}}(y)\right) \leq \varepsilon$, by Lemma 2.2, we have that $\ell\left(\left[f^{n_{k}}(x), f^{n_{k}}(y)\right]^{\mathrm{cu}}\right) \leq 2 \varepsilon$. Thus

$$
\rho \leq \ell\left([x, y]^{\mathrm{cu}}\right) \leq 2 \varepsilon \cdot \lambda_{3}^{n_{k}-1}
$$

and since $0<\lambda_{3}<1$ and $n_{k}>c \cdot n \rightarrow \infty$ we conclude that $\rho=0$ which contradicts that $x \neq y$.

Therefore we have that it is not true that for arbitrarily large $n>0$

$$
\prod_{j=1}^{n}\left\|D f^{-1} / F\left(f^{j}(y)\right)\right\| \leq \lambda_{1}^{n}
$$

and since

$$
\prod_{j=1}^{n}\left\|D f / E\left(f^{j-1}(y)\right)\right\|\left\|D f^{-1} / F\left(f^{j}(y)\right)\right\|<\lambda^{n},
$$

we may conclude from $\lambda_{1}^{2}>\lambda$ that there is $n_{0}$ such that

$$
\prod_{j=1}^{n}\left\|D f / E\left(f^{j-1}(y)\right)\right\| \leq \lambda_{1}^{n}
$$

for all $n \geq n_{0}$. Thus, in the notation of [33], $I=\left[f^{n_{0}}(x), f^{n_{0}}(y)\right]^{\text {cu }}$ is an $\left(\varepsilon, E, \lambda_{1}\right)$ interval. Let us assume, without loss of generality, that $n_{0}=0$ and so $I=[x, y]^{\text {cu }}$. 
There are two cases: either $\ell\left(f^{n}(I)\right) \rightarrow 0$ or $\ell\left(f^{n}(I)\right) \nrightarrow 0$ when $n \rightarrow \infty$. In any case we may assume that for all point $z \in I$ we have that $W_{\text {loc }}^{\text {cs }}(z)$ is a stable manifold (see [33, Corollary 3.3]) and so $W_{\mathrm{loc}}^{\mathrm{cs}}(I)$ contains a neighborhood in $M$.

Let us assume first that $\ell\left(f^{n}(I)\right) \rightarrow 0$ when $n \rightarrow \infty$. Choose $\zeta>0$ and let us find bounds for $r_{n}\left(\zeta, W_{\mathrm{loc}}^{\mathrm{cs}}(I)\right)$ where $\left.r_{n}\left(\zeta, W_{\mathrm{loc}}^{\mathrm{cs}}(I)\right)\right)$ is the minimum cardinality of a set that $(n, \zeta)$-spans $\left.W_{\text {loc }}^{\text {cs }}(I)\right)$. Since $\ell\left(f^{n}(I)\right) \rightarrow 0$ there is $n_{0}>0$ such that $\operatorname{diam}\left(f^{n}\left(W_{\text {loc }}^{\text {cs }}(I)\right)\right) \leq \zeta$ for all $n \geq n_{0}$. Hence we may find a finite subset $E$ such that $\left(\zeta, n_{0}\right)$-spans $W_{\mathrm{loc}}^{\text {cs }}(I)$ and this set also $(\zeta, n)$-spans $W_{\mathrm{loc}}^{\text {cs }}(I)$ for all $n \geq 0$. It follows readily that

$$
h\left(f, W_{\mathrm{loc}}^{\mathrm{cs}}(I), \zeta\right)=\limsup _{n \rightarrow \infty} \frac{1}{n} \log \left(r_{n}\left(\zeta, W_{\mathrm{loc}}^{\mathrm{cs}}(I)\right)\right)=0
$$

and therefore $h\left(f, W_{\mathrm{loc}}^{\mathrm{cs}}(I)\right)=0$.

On the other hand, if $\ell\left(f^{n}(I)\right) \nrightarrow 0$ then by [33, Proposition 3.1] we have that for all $z \in I$, the omega -limit set of $z, \omega(z)$, is a periodic orbit or lies in a periodic circle. (In the proof of [33, Proposition 3.1] Pujals and Sambarino assume that $f$ is of class $C^{2}$. But this is just used when $\ell\left(f^{n}(I)\right) \rightarrow 0$ in order to argue as in Schwartz's proof of the Denjoy property ([36]). If we already know that $\ell\left(f^{n}(I)\right) \nrightarrow 0$ then it is enough to assume $f$ of class $C^{1}$ to ensure that the $\omega$-limit of $I$ is contained in a periodic arc or a periodic circle.)

In case of $\omega(x)$ being included in a periodic circle $\mathcal{C}$ this circle is normally hyperbolic attracting a neighborhood $V$ of $\mathcal{C}$ and points in $V$ converge exponentially fast to $\mathcal{C}$. If $f$ is $C^{2}$ then as in [33] we conclude that the dynamics by $f^{\tau}(\tau$ being the period of $\mathcal{C}$ ) in $\mathcal{C}$ is conjugate to an irrational rotation while if $f$ is just $C^{1}$ we only have semi-conjugacy. (We may have a Cantor set as $\Omega(f / \mathcal{C})$ in $\mathcal{C}$ and wandering intervals.) In any case (conjugacy or semi-conjugacy with an irrational rotation $R_{\alpha}$ ) we profit from the fact that $h\left(R_{\alpha}\right)=0$. This implies that if $f^{\tau} / \mathcal{C}$ is conjugate or semi-conjugate to $R_{\alpha}$ then $h\left(f^{\tau} / \mathcal{C}\right)=0$.

On the other hand, if $\omega(x)$ is a periodic orbit, say, of a point $q$, since $\ell\left(f^{n}(I)\right)<\delta$ for all $n \geq 0$ we have that there is a periodic point $q^{\prime}$ in $W_{\text {loc }}^{\text {cu }}(q)$ such that attracts points in $f^{n}(I \backslash\{x\})$ (for instance the other end-point of $f^{n}(I)$ different from $f^{n}(x)$ ), see [33, Lemma 3.3.1]. Note than since $W_{\text {loc }}^{\text {cu }}(q)$ is an $\operatorname{arc}$, the period of $q^{\prime}$ is the same of that of $q$, or the double of it. Let $P$ be the set of periodic points of $f$ in $W_{\mathrm{loc}}^{\mathrm{cu}}(q) \backslash\{q\}$. Then all of them have the same period, say $\tau$. The set $P$ divides $W_{\mathrm{loc}}^{\mathrm{cu}}(q)$ in $\operatorname{arcs}$ on which the dynamics by $f^{\tau}$ is monotone. It follows that the topological entropy of $f^{\tau} / W_{\mathrm{loc}}^{\mathrm{cu}}(q)$ is zero.

So in both cases, periodic orbit or periodic circle, $f^{\tau n}\left(W_{\mathrm{loc}}^{\mathrm{cs}}(I)\right)$ approaches an $f^{\tau}$ invariant one-dimensional manifold $\mathcal{L}$ such that the topological entropy $h\left(f^{\tau}, \mathcal{L}\right)=0$. Let $\zeta>0$ and $m \in \mathbb{N}$ be given an find $S^{\prime} \subset \mathcal{L},(m, \zeta)$ spanning $\mathcal{L}$. We may find $n_{0}$ and a subset $S$ of $f^{n}(I)$ for $n \geq n_{0}$, such that $(m, \zeta)$-spans $f^{n}(I)$ with respect to $f^{\tau}$. Projecting along the fibers of the local center-stable manifolds which, by equation (1), are dynamically defined (i.e., $W_{\text {loc }}^{\text {cs }}(z)$ is a strong stable manifold for all $z \in \mathcal{L}$ ) we know that there is $n_{1}>0$ such that for any point $z \in I, \ell\left(f^{n}\left(W_{\mathrm{loc}}^{\mathrm{cs}}(z)\right)\right)<\zeta$ for 
all $n \geq n_{1}$. We add a finite number of points to $S$ in order to ensure that we have an $(m, \zeta)$ spanning set for $f^{j}\left(W_{\mathrm{loc}}^{\text {cs }}(I)\right)$ for $j=0,1, \ldots, n_{1}-1$. We conclude that $h\left(f, W_{\text {loc }}^{\text {cs }}(I), \zeta\right)=0$ since the set $S(m, \zeta)$ spans $f^{n}\left(W_{\text {loc }}^{\text {cs }}(I)\right)$ for all $n \geq 0$. Since $\zeta>0$ is arbitrary we obtain that $h\left(f, W_{\mathrm{loc}}^{\mathrm{cs}}(I)\right)=0$.

Similarly if $y \in W_{\mathrm{loc}}^{\mathrm{cs}}(x)$ then $J=[x, y]^{\mathrm{cs}}$ is an $\varepsilon-F$-interval and reasoning with the $\alpha$-limit of $J$ we obtain that $h\left(f, W_{\mathrm{loc}}^{\mathrm{cu}}(J)\right)=0$.

By [7, Corollary 2.3] we have that if there is an $\varepsilon$ - $E$-interval $I$ (or an $\varepsilon$ - $F$-interval $J)$ such that $\Gamma_{\varepsilon}(x) \subset W_{\text {loc }}^{\text {cs }}(I)$ (resp.: $\left.\Gamma_{\varepsilon}(x) \subset W_{\text {loc }}^{\text {cu }}(J)\right)$ then $h\left(\Gamma_{\varepsilon}(x), f\right)=0$. So in that case the proof of Theorem $\mathrm{A}$ is complete.

Assume now that $y \in \Gamma_{\varepsilon}(x)$ but $y \notin W_{\text {loc }}^{\mathrm{cs}}(x), y \notin W_{\text {loc }}^{\mathrm{cu}}(x)$. We will reduce this case to the previous one.

Since $y \in \hat{K}$ we have that $W_{\text {loc }}^{\text {cs }}(y)$ and $W_{\text {loc }}^{\text {cu }}(y)$ are well defined and are embedded arcs. Since for all $z \in U(K)$ the angle between $E(z)$ and $F(z)$ is bounded from below by $\gamma>0$, shrinking $\varepsilon$ if necessary, from $\operatorname{dist}(z, w) \leq \varepsilon, z, w \in \hat{K}$, we may assume firstly that $W_{\mathrm{loc}}^{\mathrm{cs}}(w) \cap W_{\mathrm{loc}}^{\mathrm{cu}}(z)=w_{F}$ and $W_{\mathrm{loc}}^{\mathrm{cs}}(z) \cap W_{\mathrm{loc}}^{\mathrm{cu}}(w)=w_{E}$, and secondly that

$$
\begin{aligned}
f\left(w_{F}\right) & =W_{\mathrm{loc}}^{\mathrm{cs}}(f(w)) \cap W_{\mathrm{loc}}^{\mathrm{cu}}(f(z)), \\
f\left(w_{E}\right) & =W_{\mathrm{loc}}^{\mathrm{cu}}(f(w)) \cap W_{\mathrm{loc}}^{\mathrm{cs}}(f(z)), \\
f^{-1}\left(w_{F}\right) & =W_{\mathrm{loc}}^{\mathrm{cs}}\left(f^{-1}(w)\right) \cap W_{\mathrm{loc}}^{\mathrm{cu}}\left(f^{-1}(z)\right), \\
f^{-1}\left(w_{E}\right) & =W_{\mathrm{loc}}^{\mathrm{cu}}\left(f^{-1}(w)\right) \cap W_{\mathrm{loc}}^{\mathrm{cs}}\left(f^{-1}(z)\right) .
\end{aligned}
$$

Letting $z=x, w=y$, we obtain points $\left.y_{F}=W_{\mathrm{loc}}^{\mathrm{cs}}(y)\right) \cap W_{\mathrm{loc}}^{\mathrm{cu}}(x)$ and $y_{E}=W_{\mathrm{loc}}^{\mathrm{cs}}(x) \cap$ $W_{\text {loc }}^{\text {cu }}(y)$. Since $y \notin W_{\text {loc }}^{\text {cs }}(x) \cup W_{\text {loc }}^{\text {cu }}(x)$ it holds that $y_{E} \neq x$ and $y_{F} \neq x$. See figure 5 .

Since $\operatorname{dist}\left(f^{n}(x), f^{n}(y)\right) \leq \varepsilon$ for all $n \in \mathbb{Z}$, by induction we have that

$$
f^{n}\left(y_{F}\right)=W_{\mathrm{loc}}^{\mathrm{cs}}\left(f^{n}(y)\right) \cap W_{\mathrm{loc}}^{\mathrm{cu}}\left(f^{n}(x)\right) \text { for all } n \in \mathbb{Z} \text {. }
$$

Moreover, we have that

$$
\operatorname{dist}\left(f^{n}(x), f^{n}\left(y_{F}\right)\right)<\frac{3 \operatorname{dist}\left(f^{n}(x), f^{n}(y)\right)}{\sin \gamma}<\frac{3 \varepsilon}{\sin \gamma}<\frac{\delta_{3}}{2},
$$

which implies by Lemma 2.2 that $\ell\left(f^{n}\left(\left[x, y_{F}\right]^{\mathrm{cu}}\right)=\ell\left(\left[f^{n}(x), f^{n}\left(y_{F}\right)\right]^{\mathrm{cu}}\right)<\delta_{3}\right.$, which in turn implies that $\operatorname{dist}\left(f^{n}(x), f^{n}(z)\right)<\delta_{3}$ for all $z \in\left[x, y_{F}\right]^{\text {cu }}$ and for all $n \geq 0$.

Thus $\left[x, y_{F}\right]^{\mathrm{cu}}=I$ is a $\left(\delta_{3}, E\right)$-interval (see [33]) and therefore we have that $W_{\text {loc }}^{\text {cs }}(I)$ is a stable manifold which implies that $\operatorname{dist}\left(f^{n}\left(y_{F}\right), f^{n}(y)\right) \rightarrow 0$ when $n \rightarrow \infty$. Reasoning as in the case in which $y \in W_{\text {loc }}^{\text {cu }}(x)$ we obtain

$$
h\left(f, W_{\mathrm{loc}}^{\mathrm{cs}}(I), \zeta\right)=\limsup _{n \rightarrow \infty} \frac{1}{n} \log \left(r_{n}\left(\zeta, W_{\mathrm{loc}}^{\mathrm{cs}}(I)\right)\right)=0 .
$$

Hence $h\left(f, W_{\text {loc }}^{\text {cs }}(I)\right)=0$ and from $\left.\Gamma_{\varepsilon}(x) \subset W_{\text {loc }}^{\text {cs }}(I)\right)$ we conclude that $h\left(f, \Gamma_{\varepsilon}(x)\right)=0$. Since this last inequality holds for all $x \in K$ we have that $f / K$ is $h$-expansive, finishing the proof of Theorem A. 


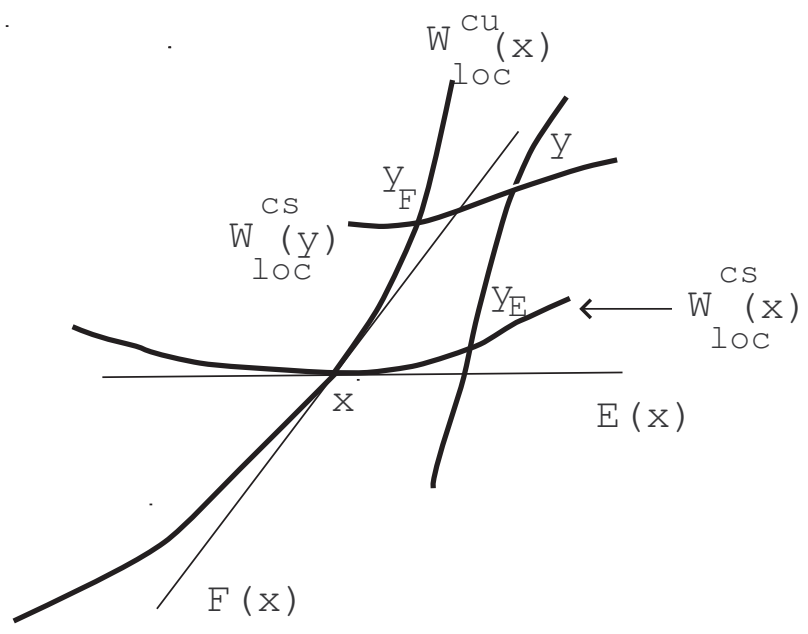

Figure 5 - Case $y \notin W_{\mathrm{loc}}^{\mathrm{cs}}(x), y \notin W_{\mathrm{loc}}^{\mathrm{cu}}(x)$.

\section{Generalization to higher dimensions}

Theorem B is a sort of generalization of Theorem A under certain restrictions. In order to state these restrictions, we recall the concept of finest dominated splitting introduced in [3].

Definition 3.1. Let $\Lambda \subset M$ be a compact $f$-invariant subset such that $T M / \Lambda=$ $E_{1} \oplus E_{2} \oplus \cdots \oplus E_{k}, E_{j}$ being $D f$ invariant, $j=1, \ldots, k$. We say that $E_{1} \oplus E_{2} \oplus \cdots \oplus E_{k}$ is dominated if for all $1 \leq j \leq k-1$

$$
\left(E_{1} \oplus \cdots \oplus E_{j}\right) \oplus\left(E_{j+1} \oplus \cdots \oplus E_{k}\right)
$$

has a dominated splitting We say that $E_{1} \oplus E_{2} \oplus \cdots \oplus E_{k}$ is the finest dominated splitting when for all $j=1, \ldots, k$ there is no possible decomposition of $E_{j}$ as two invariant sub-bundles having domination.

$C^{1}$-generically, the finest dominated splitting has a very special form.

Generic Assumptions (see $[1, \S 2.1])$

There exists a residual subset $\mathcal{G}$ of $\operatorname{Diff}^{1}(M)$ such that if $f: M \rightarrow M$ is a diffeomorphisms belonging to $\mathcal{G}$ then

(i) $f$ is Kupka-Smale, (i.e., all periodic points are hyperbolic and their stable and unstable manifolds intersect transversally). 
(ii) For any pair of saddles $p, q$, either $H(p, f)=H(q, f)$ or $H(p, f) \cap H(q, f)=\emptyset$.

(iii) For any saddle $p$ of $f, H(p, f)$ depends continuously on $g \in \mathcal{G}$.

(iv) The periodic points of $f$ are dense in $\Omega(f)$.

(v) The chain recurrent classes of $f$ form a partition of the chain recurrent set of $f$.

(vi) Every chain recurrent class containing a periodic point $p$ is the homoclinic class associated to that point.

Theorem 3.2. There is a residual subset $\mathcal{I} \subset \mathcal{G}$ of $\operatorname{Diff}^{1}(M)$ such that if $f \in \mathcal{I}$ has a homoclinic class $H(p, f)$ which contains hyperbolic saddles of indices $i<j$ then either

(i) For any neighborhood $U$ of $H(p, f)$ and any $C^{1}$-neighborhood $\mathcal{U}$ of $f$ there is a diffeomorphism $g \in \mathcal{U}$ with a homoclinic tangency associated to a saddle of the homoclinic class $H\left(p_{g}, g\right)$, where $p_{g}$ is the continuation of $p$.

(ii) There is a dominated splitting

$$
T_{H(p, f)} M=E \oplus F_{1} \oplus \cdots \oplus F_{j-i} \oplus G
$$

with $\operatorname{dim}(E)=i$ and $\operatorname{dim}\left(F_{h}\right)=1$ for all $h$ and $\operatorname{dim}(G)=\operatorname{dim}(M)-j$. Moreover, the sub-bundles $F_{h}$ are not hyperbolic.

Proof. This is [1, Corollary 3] taking into account that a result by Gourmelon guarantees that the homoclinic tangency can be associated to a saddle inside the homoclinic class (see [19, Corollary, 6.6.2, Theorem 6.6.8]).

Remark 3.3. In Theorem 3.2 we cannot assure that $E$ is contracting and $G$ is expanding unless the homoclinic class is isolated (see $[1,4]$ ).

Sketch of the proof of Theorem B. Observe first that by assumption we are $C^{1}$-far from homoclinic tangencies. Therefore taking into account Theorem 3.2 and assuming that $H(p, f)$ is isolated, we have that for all $x \in H(p, f)$ it holds that

$$
T_{x} M=E_{0}(x) \oplus E_{1}(x) \oplus \cdots \oplus E_{k}(x) \oplus E_{k+1}(x),
$$

with $E_{0}(x)$ contracting, $E_{k+1}(x)$ expanding and all $E_{j}(x), j=1, \ldots, k$, one dimensional and not hyperbolic. From this result we have that the proof of Theorem B is similar to the proof of Theorem A. Let $y \in \Gamma_{\varepsilon}(x)$ where $x \in H(p, f)$. We cannot have $y \in W_{\text {loc }}^{u}(x)$, where $W_{\text {loc }}^{u}(x)$ is the local unstable manifold tangent to $E_{k+1}$. Otherwise, since on $E_{k+1} D f$ acts as a hyperbolic expansion, we will have that for some $n_{0}>0$ it holds that $\operatorname{dist}\left(f^{n_{0}}(x), f^{n_{0}}(y)\right)>\varepsilon$. If we choose $\varepsilon>0$ less than the minimum of the diameters of the local unstable manifolds of points in $H(p, f)$, then we arrive to a contradiction. Similarly we cannot have that there is 
$y \in \Gamma_{\varepsilon}(x) \cap W_{\text {loc }}^{s}(x)$ where $W_{\text {loc }}^{s}(x)$ is the local stable manifold tangent to $E_{0}$. Suppose now that $y \notin W_{\mathrm{loc}}^{s}(x) \cup W_{\mathrm{loc}}^{u}(x)$. If $y \in \Gamma_{\varepsilon}(x)$ we may project $f^{n}(y)$ along a center stable manifold tangent to $E_{0}\left(f^{n}(y)\right) \oplus E_{1}\left(f^{n}(y)\right) \oplus \cdots \oplus E_{k}\left(f^{n}(y)\right)$ into the unstable manifold of $f^{n}(x)$ obtaining a point $f^{n}\left(y^{\prime}\right) \in W_{\mathrm{loc}}^{u}\left(f^{n}(x)\right)$. (These centerstable manifolds are just locally defined but since $\operatorname{dist}\left(f^{n}(x), f^{n}(y)\right) \leq \varepsilon \forall n \in \mathbb{Z}$, by [33, Lemma 3.0.4, Corollary 3.2], as in equation (2) we have that, for all $n \in \mathbb{Z}$, $f^{n}\left(y^{\prime}\right)=W_{\mathrm{loc}}^{\mathrm{cs}}\left(f^{n}(y)\right) \cap W_{\mathrm{loc}}^{u}\left(f^{n}(x)\right)$.) Since the angles between the different directions $E_{j}$ given by the dominated splitting are bounded away from zero the diameter of this projection goes to zero when $\varepsilon$ goes to zero. So this projection $f^{n}\left(y^{\prime}\right)$ belongs to $\Gamma_{L \varepsilon}\left(f^{n}(x)\right)$ for some constant $L>0$ which depends on the lower bound for the angles between the different sub-bundles of the splitting. Thus, as in the proof of Theorem A, we obtain that the projection $y^{\prime}$ coincides with $x$. Similarly the projection of $y$ along a center unstable manifold tangent to $E_{1}(y) \oplus \cdots \oplus E_{k+1}(y)$ into the stable manifold of $x$ (tangent to $E_{0}(x)$ ) has to coincide with $x$ for sufficiently small $\varepsilon>0$. Therefore $\Gamma_{\varepsilon}(x)$ is included in a center manifold of $x, W_{\mathrm{loc}}^{c}(x)$. Either $W_{\mathrm{loc}}^{c}(x)$ is one-dimensional $(k=1)$ and then we may argue as in the first part of Theorem A, or there is some surface $S$ tangent to $E_{j}(x), E_{j+1}(x)$ for some $j \in\{1, \ldots, k-1\}$ in which there is a projection $y^{\prime} \neq x$ of $y$ along a center unstable manifold tangent to $E_{j+2}(y), \ldots, E_{k+1}(y)$ such that $y^{\prime} \in \Gamma_{K \varepsilon}(x)$. In the former case the existence of $y^{\prime}$ implies readily that there is $n_{0} \geq 0$ such that $E_{j+2}\left(f^{n}\left(y^{\prime}\right)\right) \oplus \cdots \oplus E_{k+1}\left(f^{n}\left(y^{\prime}\right)\right)$ is uniformly expanding for all $n \geq n_{0}$ and that $E_{0}\left(f^{n}\left(y^{\prime}\right)\right) \oplus \cdots \oplus E_{j-1}\left(f^{n}\left(y^{\prime}\right)\right)$ is uniformly contracting for $n \leq-n_{0}$. Therefore points in $\Gamma_{\varepsilon}(x)$ have to be in $S$. Hence we may repeat the second part of the proof of Theorem A taking into account that $E_{i}$ is one dimensional for all $i=1, \ldots, k$. In any case we obtain that $h_{\text {top }}\left(\Gamma_{\varepsilon}(x)\right)=0$. Therefore $f / H(p, f)$ is entropy expansive. This finishes the sketch of the proof.

\section{Robust $h$-expansiveness implies domination on surfaces}

In this section we prove Theorem C. In order to do that we will argue by contradiction assuming that we do not have a dominated splitting. This will allow us to create a tangency between the stable and unstable manifolds of $p$. Using results of Downarowicz and Newhouse (see [15] and [28]) we will see that this is not possible when $f / H(p)$ is $h$-expansive in a robust way.

Recall that a subshift $(g, Y)$ is the restriction of the full shift in a finite alphabet to a closed invariant subsystem.

Definition 4.1. Let $f: X \rightarrow X$ be a homeomorphism of a compact metric space $X$. A symbolic extension of the pair $(f, X)$ is a subshift $(g, Y)$ with a continuous surjection $\pi: Y \rightarrow X$ such that $f \pi=\pi g$. Let us define for any $f$-invariant measure $\mu$, $h_{\text {ext }}^{\pi}(\mu)=\sup \left\{h_{\nu}(g): \pi_{*} \nu=\mu\right\}$. An extension $(g, Y)$ is principal if it preserves the measure theoretic entropy $h_{\mu}(f)$ of the factor $(f, X)$ for any $f$-invariant measure $\mu$, $h_{\text {ext }}^{\pi}(\mu)=h_{\mu}(f)$. In particular if a symbolic extension is principal then the topological entropy of the extension coincides with that of the original system, that is, 
$h(g, Y)=h(f, X)$.

Theorem 4.2 (Downarowicz, Newhouse). Fix $2 \leq r<\infty$. There is a residual subset $\mathcal{R}$ of the space $\operatorname{Diff}^{r}(M)$ of $C^{r}$-diffeomorphisms of a closed surface $M$ such that if $f \in \mathcal{R}$ and $f$ has a homoclinic tangency, then $f$ has no principal symbolic extension.

Proof. See [15, Theorem 1.4].

Moreover, if $f$ has no principal symbolic extension then $f$ cannot be asymptotically $h$-expansive as has been proved by M. Boyle, D. Fiebig, and U. Fiebig (see [6]).

Proof of Theorem $C$. Let $H(p)$ be a non trivial $f$-homoclinic class associated to the hyperbolic periodic point $p$. Assume that there is a $C^{1}$ neighborhood $\mathcal{U}$ of $f$ such that for any $g \in \mathcal{U}$ it holds that there is a continuation $H\left(p_{g}\right)$ of $H(p)$ such that $H\left(p_{g}\right)$ is $h$-expansive. Let $x \in W^{s}(p) \cap W^{u}(p)$ be a transverse homoclinic point associated to the periodic point $p$. We define $E(x) \equiv T_{x} W^{s}(p)$ and $F(x) \equiv T_{x} W^{u}(p)$. Since $p$ is hyperbolic we have that $E(x) \oplus F(x)=T_{x} M$. Moreover, $E(x)$ and $F(x)$ are $D f$-invariant, i.e., $D f(E(x))=E(f(x))$ and $D f(F(x))=F(f(x))$.

By definition $H(p) \equiv \operatorname{clos}(\operatorname{hom}(p))$ where hom $(p)$ is the set of transverse homoclinic points associated to $p$ so if we prove that there is a dominated splitting for $\operatorname{hom}(p)$ we are done since then we can extend by continuity the splitting to the closure $H(p)$. Moreover, since $C^{2}$-diffeomorphisms are dense in the $C^{1}$-neighborhood $\mathcal{U}$ we may assume that $f$ is of class $C^{2}$.

So let us assume that $f$ is of class $C^{2}$ and prove that there is a dominated splitting for $\operatorname{hom}(p)$. To do so it is enough to prove that there exists $m>0$ such that for some $k: 0 \leq k \leq m$ it holds for all $x \in \operatorname{hom}(p)$ that

$$
\left\|D f^{k} / E(x)\right\|\left\|D f^{-k} / F\left(f^{k}(x)\right)\right\| \leq \frac{1}{2} .
$$

Hence arguing by contradiction let us assume that for all $m>0$ there is $x_{m} \in \operatorname{hom}(p)$ such that for all $k$ with $0 \leq k \leq m$ we have

$$
\left\|D f^{k} / E\left(x_{m}\right)\right\|\left\|D f^{-k} / F\left(f^{k}\left(x_{m}\right)\right)\right\|>\frac{1}{2} .
$$

Hence, as in $[24,35]$, given any $\gamma>0$ and $\varepsilon>0$ we may find $m>0$, depending on $\varepsilon$ and $\gamma$, such that with an $\varepsilon-C^{1}$-perturbation we obtain a $C^{2}$ diffeomorphism $g^{\prime}$ with a homoclinic point $x_{g^{\prime}}$ associated to $p_{g^{\prime}}$ such that the angle at $x_{g^{\prime}}$ between $W_{\text {loc }}^{s}\left(x_{g^{\prime}}, g^{\prime}\right)$ and $W_{\text {loc }}^{u}\left(x_{g^{\prime}}, g^{\prime}\right)$ is less than $\gamma$. Since $\gamma$ is arbitrarily small we may $C^{1}$-perturb $g^{\prime}$ obtaining $g^{\prime \prime}$ of class $C^{2}$ with a tangency at $x_{g^{\prime \prime}}$ between $W_{\text {loc }}^{s}\left(x_{g^{\prime \prime}}\right)$ and $W_{\mathrm{loc}}^{u}\left(x_{g^{\prime \prime}}\right)$. As in [27] we may $C^{2}$-perturb $g^{\prime \prime}$ obtaining $g$ in $\operatorname{Diff}^{2}(M)$ with a $C^{2}$ robust tangency of Hènon-like type. By the results of $[15]$ and [28] we conclude that there is no symbolic extension for $g / H\left(p_{g}\right)$. Therefore, by [6], $g / H\left(p_{g}\right)$ is not asymptotically $h$-expansive and $a$ fortior $i$ it is not $h$-expansive contradicting our hypotheses. This finishes the proof of Theorem C. 


\section{References}

[1] F. Abdenur, Ch. Bonatti, S. Crovisier, L. J. Díaz, and L. Wen, Periodic points and homoclinic classes, Ergodic Theory Dynam. Systems 27 (2007), no. 1, 1-22.

[2] C. Bonatti and S. Crovisier, Récurrence et généricité, Invent. Math. 158 (2004), no. 1, 33-104 (French, with English and French summaries).

[3] C. Bonatti, L. J. Díaz, and E. R. Pujals, A $C^{1}$-generic dichotomy for diffeomorphisms: Weak forms of hyperbolicity or infinitely many sinks or sources, Ann. of Math. (2) 158 (2003), no. 2, 355-418.

[4] C. Bonatti, L. J. Díaz, E. R. Pujals, and J. Rocha, Robustly transitive sets and heterodimensional cycles, Astérisque 286 (2003), 187-222.

[5] C. Bonatti, L. J. Díaz, and M. Viana, Dynamics beyond uniform hyperbolicity, Encyclopaedia of Mathematical Sciences, Mathematical Physicx, vol. 102, Springer-Verlag, Berlin, 2005.

[6] M. Boyle, D. Fiebig, and U. Fiebig, Residual entropy, conditional entropy and subshift covers, Forum Math. 14 (2002), no. 5, 713-757.

[7] R. Bowen, Entropy-expansive maps, Trans. Amer. Math. Soc. 164 (1972), 323-331.

[8] J. Buzzi, Intrinsic ergodicity of smooth interval maps, Israel J. Math. 100 (1997), 125-161.

[9] C. M. Carballo, C. A. Morales, and M. J. Pacifico, Homoclinic classes for generic $C^{1}$ vector fields, Ergodic Theory Dynam. Systems 23 (2003), no. 2, 403-415.

[10] S. Crovisier, Periodic orbits and chain-transitive sets of $C^{1}$-diffeomorphisms, Publ. Math. Inst. Hautes Études Sci. 104 (2006), 87-141.

[11] L. J. Díaz, Robust nonhyperbolic dynamics and heterodimensional cycles, Ergodic Theory Dynam. Systems 15 (1995), no. 2, 291-315.

[12] _ Persistence of cycles and nonhyperbolic dynamics at heteroclinic bifurcations, Nonlinearity 8 (1995), no. 5, 693-713.

[13] L. J. Díaz, E. R. Pujals, and R. Ures, Partial hyperbolicity and robust transitivity, Acta Math. 183 (1999), no. 1, 1-43.

[14] L. J. Díaz and J. Rocha, Partially hyperbolic and transitive dynamics generated by heteroclinic cycles, Ergodic Theory Dynam. Systems 21 (2001), no. 1, 25-76.

[15] T. Downarowicz and S. Newhouse, Symbolic extensions and smooth dynamical systems, Invent. Math. 160 (2005), no. 3, 453-499.

[16] J. Franks, Necessary conditions for stability of diffeomorphisms, Trans. Amer. Math. Soc. 158 (1971), 301-308.

[17] M. Gerber, Conditional stability and real analytic pseudo-Anosov maps, Mem. Amer. Math. Soc. 54 (1985), no. 321, iv+116.

[18] M. Gerber and A. Katok, Smooth models of Thurston's pseudo-Anosov maps, Ann. Sci. École Norm. Sup. (4) 15 (1982), no. 1, 173-204.

[19] N. Gourmelon, Instabilité de la dynamique en l'absence de décomposition dominée, Thèse de doctorat, Université de Bourgogne, 2007.

[20] S. Hayashi, Connecting invariant manifolds and the solution of the $C^{1}$ stability and $\Omega$-stability conjectures for flows, Ann. of Math. (2) 145 (1997), no. 1, 81-137.

[21] A. Katok, Lyapunov exponents, entropy and periodic orbits for diffeomorphisms, Inst. Hautes Études Sci. Publ. Math. 51 (1980), 137-173.

[22] J. Lewowicz and E. Lima de Sá, Analytic models of pseudo-Anosov maps, Ergodic Theory Dynam. Systems 6 (1986), no. 3, 385-392. 
[23] R. Mañé, Expansive diffeomorphisms, Dynamical systems (Warwick, 1974), Lecture Notes in Math., vol. 468, Springer, Berlin, 1975, pp. 162-174.

[24] _ An ergodic closing lemma, Ann. of Math. (2) 116 (1982), no. 3, 503-540.

[25] A proof of the $C^{1}$ stability conjecture, Inst. Hautes Études Sci. Publ. Math. 66 (1988), $161-210$.

[26] M. Misiurewicz, Diffeomorphisms without any measure with maximal entropy, Bull. Acad. Polon. Sci. Sér. Sci. Math. Astronom. Phys. 21 (1973), 903-910.

[27] S. E. Newhouse, The abundance of wild hyperbolic sets and nonsmooth stable sets for diffeomorphisms, Inst. Hautes Études Sci. Publ. Math. 50 (1979), 101-151.

[28] _ New phenomena associated with homoclinic tangencies, Ergodic Theory Dynam. Systems 24 (2004), no. 5, 1725-1738.

[29] M. J. Pacifico, E. R. Pujals, M. Sambarino, and J. L. Vieitez, Robustly expansive codimensionone homoclinic classes are hyperbolic, Ergodic Theory Dynam. Systems, to appear.

[30] M. J. Pacifico, E. R. Pujals, and J. L. Vieitez, Robustly expansive homoclinic classes, Ergodic Theory Dynam. Systems 25 (2005), no. 1, 271-300.

[31] V. A. Pliss, Analysis of the necessity of the conditions of Smale and Robbin for structural stability for periodic systems of differential equations, Differencial'nye Uravnenija 8 (1972), 972-983 (Russian).

[32] C. C. Pugh, The closing lemma, Amer. J. Math. 89 (1967), 956-1009.

[33] E. R. Pujals and M. Sambarino, Homoclinic tangencies and hyperbolicity for surface diffeomorphisms, Ann. of Math. (2) 151 (2000), no. 3, 961-1023.

[34] - On the dynamics of dominated splitting, preprint, available at http://premat.fing. edu.uy (premat2003/70).

[35] M. Sambarino and J. L. Vieitez, On $C^{1}$-persistently expansive homoclinic classes, Discrete Contin. Dyn. Syst. 14 (2006), no. 3, 465-481.

[36] A. J. Schwartz, A generalization of a Poincaré-Bendixson theorem to closed two-dimensional manifolds., Amer. J. Math. 85 (1963), 453-458; errata, ibid., Amer. J. Math. 85 (1963), 753.

[37] S. Smale, Differentiable dynamical systems, Bull. Amer. Math. Soc. 73 (1967), 747-817. 